\title{
A Requirements-Driven Optimization Method for Acoustic Treatment Design
}

\author{
Jeffrey J. Berton* \\ NASA Glenn Research Center, Cleveland, Ohio 44135
}

\begin{abstract}
Acoustic treatment designers have long been able to target specific noise sources inside turbofan engines. Facesheet porosity and cavity depth are key design variables of perforateover-honeycomb liners that determine levels of noise suppression as well as the frequencies at which suppression occurs. Layers of these structures can be combined to create a robust attenuation spectrum that covers a wide range of frequencies. Looking to the future, rapidlyemerging additive manufacturing technologies are enabling new liners with multiple degrees of freedom, and new adaptive liners with variable impedance are showing promise. More than ever, there is greater flexibility and freedom in liner design. Subject to practical considerations, liner design variables may be manipulated to achieve a target attenuation spectrum. But characteristics of the ideal attenuation spectrum can be difficult to know. Many multidisciplinary system effects govern how engine noise sources contribute to community noise. Given a hardwall fan noise source to be suppressed, and using an analytical certification noise model to compute a community noise measure of merit, the optimal attenuation spectrum can be derived using multidisciplinary systems analysis methods. The subject of this paper is an analytical method that derives the ideal target attenuation spectrum that minimizes noise perceived by observers on the ground.
\end{abstract}

Nomenclature

$\begin{array}{lll}=\text { source noise modeling constant } & x & =\text { suppression spectrum shape factor } \\ =\text { enclosed area of suppression spectrum } & \theta & =\text { polar (yaw) emission angle, zero at inlet } \\ =\text { frequency } & \text { Subscripts: } & \\ =\text { convective amplification term } & b & =\text { broadband } \\ =\text { noise level } & \text { ex } & =\text { fan exit } \\ =\text { Mach number } & f & =\text { flight } \\ =\text { fan shaft rotational speed } & i & =\text { index counter } \\ =\text { optimization objective function } & \text { in } & =\text { fan inlet } \\ =\text { dynamic constraint multiplier } & s & =\text { suppression } \\ =\text { weighting factor } & t & =\text { tone }\end{array}$

\section{Introduction}

$\mathrm{T}$

HE most common type of acoustic treatment found in modern turbofan engine inlets and bypass exhaust ducts are resistive facesheet resonators. Resonators generate destructive interference that reduces the level of noise propagating through a duct. A conventional resonator consists of a perforated sheet laid over cavities constructed from a honeycomb core. These perforate-over-honeycomb liners are preferred in practice over bulk absorbers due to their suitability for use in harsh operational environments. Subject to practical structural, maintenance and environmental considerations, liner design variables can be manipulated to achieve a target attenuation spectrum. Principal liner design variables are facesheet porosity (determining the absorption peak level) and the honeycomb cavity depth (determining the absorption peak frequency). One, two, or even three layers of these structures are found in practice. Layers are combined to create a robust attenuation spectrum that covers a wide range of frequencies. A schematic of a single degree of freedom perforate-over-honeycomb liner is shown in Figure 1.

\footnotetext{
*Aerospace Engineer, Propulsion Systems Analysis Branch, MS 5-11, senior member AIAA.
} 
Looking to the future, industrial innovations such as additive manufacturing are enabling new liners with multiple degrees of freedom. Cavities of variable geometry can be built up from a rigid backplate using additive techniques. Complex cavities consisting of tunnels with straight segments and sharp turns can be constructed that are not feasible using conventional manufacturing processes.

New adaptive and active liners are being investigated as well. Since the acoustic signature of a fan changes with engine power setting and flight condition, the noise source that liner designers aim to suppress is effectively a moving target. A dynamically-changing source may be more effectively suppressed with active and adaptive liners. New, shape-memory materials and other techniques are enabling morphing, more-tunable liner geometries that can adjust to a changing noise source by tailoring its suppression spectrum.

To the point, today there is greater flexibility and freedom in liner design. More than ever, liner design variables can be manipulated to achieve a target attenuation spectrum. But characteristics of the ideal attenuation spectrum can be difficult to know. Hardwall fan noise

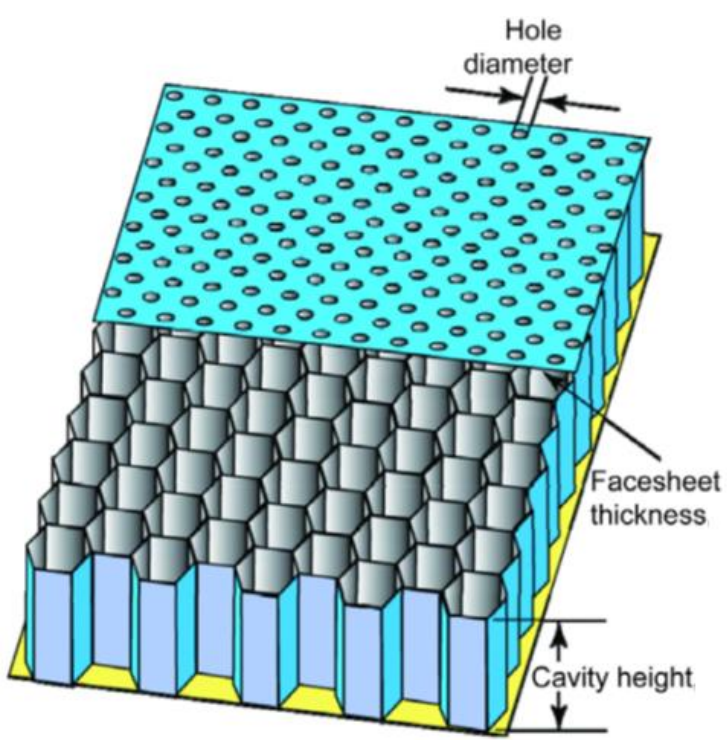

Figure 1. Single degree of freedom, perforate-overhoneycomb liner (Graphic reproduced from Ref. 1). character varies dramatically with engine operation, size, architecture and design intent. The "best" liner attenuation spectrum varies by application with the metrics used to measure it.

The ideal liner perhaps may be one that can be tuned to minimize community noise measured at the three certification locations (governed by ICAO's Annex $16^{2}$, or its FAA equivalent, Part $36^{3}$ ). Propagation of an engine noise source to an observer on the ground is a complex physical phenomenon, and the process of measuring it with relevant metrics while abiding by airworthiness regulations adds more complexity. Many multidisciplinary system effects govern how engine noise sources contribute to community noise. Thus, accurately predicting community noise using analytical methods is a daunting prospect. Despite this, aircraft system noise prediction is an analytically tractable problem.

If the shape of a liner suppression spectrum can be mathematically parameterized, then a set of independent "shape factor" design variables can be used to express suppression levels as a function of frequency. Given a hardwall noise source to suppress, it should then be straightforward to manipulate the shape factor variables using an optimizer until community noise is minimized. Once the ideal suppression spectrum is known, the geometric design and the impedance characteristics of a real acoustic liner could be derived to match it. In other words, the optimum acoustic liner design can be approached using a requirements-driven process to assist the traditional "bottoms-up" design.

The subject of this paper is an analytical method that derives the ideal target attenuation spectrum that minimizes noise perceived by observers on the ground. Software is developed to test and validate the method on a simple, notional source. With experience gained from minimizing noise of the simple source, the method is tested against a realistic and complex source.

\section{Method of Analysis}

\section{A. System Noise Modeling}

Analytically modeling airport community noise is a complex proposition. In most systems-level prediction methods, aircraft noise sources are typically modeled using free-field, lossless sound pressure levels defined on an arc of constant radius. In the case of fan noise, the source is usually treated as compact, at least insofar as a distant observer is concerned. The noise levels are cast as a function of frequency, emission angle(s), flight condition, and engine state. This modeling process is shown schematically in Figure 2. The noise source subject to liner suppression can be combined with other sources nearby on the airplane or modified by other local effects before propagation to the ground. As the source is analytically flown through the air, its acoustic signature changes. From the viewpoint of a stationary observer, distance and emission angles vary as the source first approaches and then recedes. Doppler shift and convective amplification alter levels and pitch observed on the ground. As the emissions propagate, they are influenced by spherical spreading, atmospheric absorption, and various ground effects.

\section{2 of 12}

American Institute of Aeronautics and Astronautics 


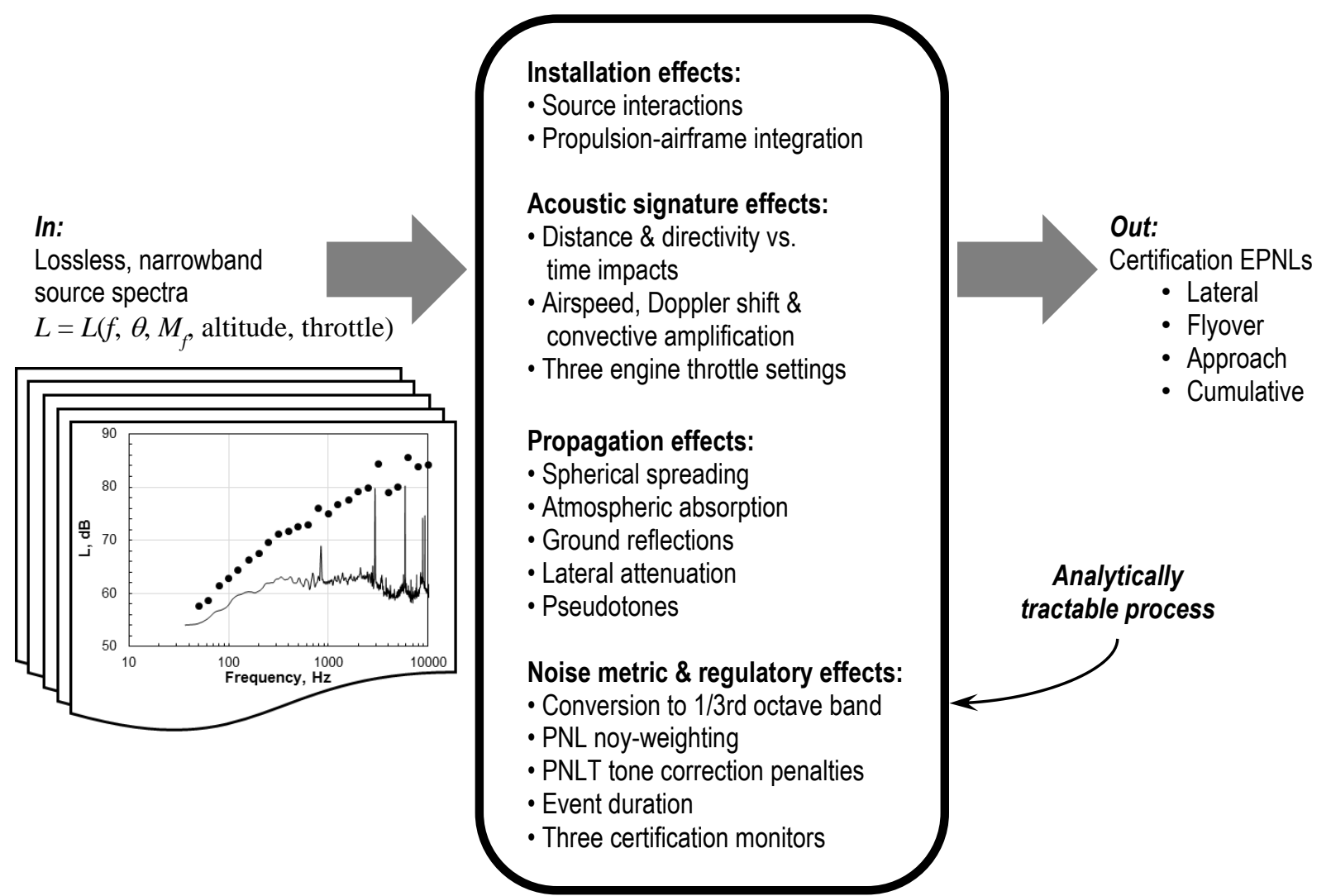

Figure 2. System noise prediction schematically represented as an analytical modeling process.

If noise certification metrics for jet airplanes are used as a measure of merit, additional complexities arise. Airworthiness certification attempt to account for various psychoacoustic phenomena. Noise regulations are based upon the one-third octave band center frequency paradigm. Received noise levels are converted to perceived noise levels using noy-weighting factors. Particular attention is given to levels in the frequencies between $1000 \mathrm{~Hz}$ and $10,000 \mathrm{~Hz}$. An additional noise penalty is added if tones are present in the received spectra, with the highest penalties assigned to tones lying between $500 \mathrm{~Hz}$ and $5000 \mathrm{~Hz}$.

During a noise certification test, spectral acoustic measurements are made as an airplane flies past three certification noise observation monitors on the ground (shown in Figure 3). Spectra are measured at half-second time intervals at each noise observation station. From these, tone-corrected perceived noise levels are computed.

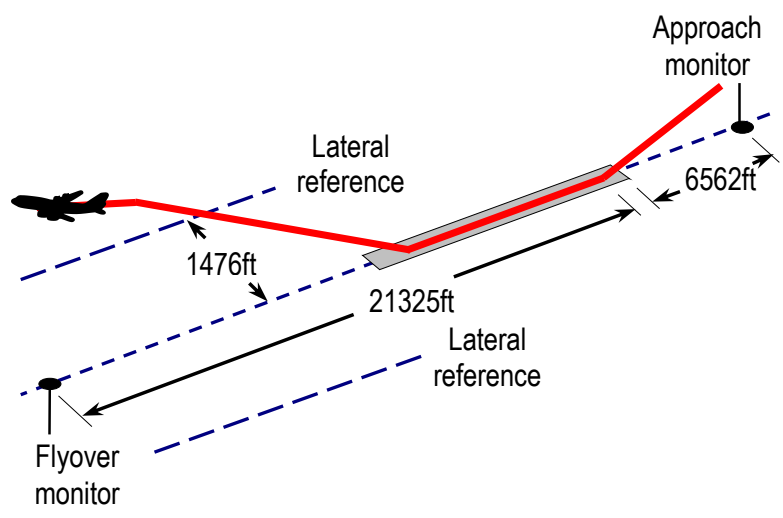

\section{Figure 3. Noise certification monitor arrangement} relative to takeoff and landing flight paths.

For transport-category large airplanes and for subsonic jet-powered airplanes of all sizes, the regulation metrics are cast in terms of the Effective Perceived Noise Level, or EPNL. The EPNL is an attempt to relate human annoyance to noise. It is a metric sensitive to frequency, tone content, and duration of a single airplane flyover event. It is the value regulated by ICAO and by the FAA for noise type certification. In noise certification parlance, the cumulative, or algebraic, sum of the three certification EPNLs is often used to capture all three measurements.

The community noise modeling process illustrated in Figure 2 is an analytically tractable problem, solvable by physics-based system analysis tools. NASA's Aircraft Noise Prediction Program (ANOPP ${ }^{4,5}$ ) is an example of such a tool. In this study, ANOPP is given a noise source (suppressed by acoustic treatment), analytically flies it through the air, propagates it to an observer on the ground, and computes EPNLs.

3 of 12

American Institute of Aeronautics and Astronautics 


\section{B. Candidate Suppression Models}

The suppression spectra are parameterized mathematically using a set of independent design variables. These "shape factor" variables are subject to manipulation by a multi-variable optimizer so that an objective function representing community noise is minimized. The shape factors are represented by a vector, $\mathbf{x}$, consisting of elements $x_{i}$. By this definition, a suppression spectrum can be represented by any number of simple mathematical expressions, as long as they faithfully characterize the shape of an actual suppression spectrum. A parameterized suppression spectrum is, in other words, a simplified metamodel or surrogate model of the actual suppression spectrum.

A simple example of a parameterized suppression spectrum might be given by a single-variable polynomial function of narrowband frequency. The narrowband linear bandwidth of frequency is arbitrary, as long as there is sufficient detail to describe the spectrum. Frequency could range from zero to $11,220 \mathrm{~Hz}$ : the upper boundary of the one-third octave band center frequency defined by aviation regulations. ${ }^{6}$ The coefficients of the polynomial would be the shape factor variables $x_{i}$, subject to optimization. At each pass of the optimizer, the calculated suppression would be subtracted from the known, untreated source, creating a suppressed source. Its levels would be converted to the one-third octave band, analytically flown through the air, propagated to the ground, and an appropriate objective function would be computed.

Discretized suppression spectra are also possible. A suppression spectrum can be defined by discrete training points connected by spline functions. If their frequencies are defined, their levels become the $x_{i}$ to be varied by the optimizer. If many training points are used, shape control can be very precise, at the risk of burdening the optimizer with many independent variables. Of course, most classical and evolutionary optimizers tend to perform best when there are as few design variables as possible. When dealing with discretized suppression spectra, a simplifying tactic is to enumerate values for the $x_{i}$ rather than let them be continuous. Evolutionary optimizers are quite good in dealing with enumerated variables.

But suppression levels need to be limited somehow, otherwise the "best" shape factors will be those that deliver ridiculously large and unrealistic levels of suppression. Unconstrained, an optimizer would drive the $x_{i}$ of, say, a discretized spectrum to very large values. One way to limit suppression is to require a constant area be enclosed by its spectrum. In other words, as shape factors $x_{i}$ are varied and optimized, the area bounded by the curve of the suppression spectrum over the frequency domain is required to remain constant. There is some physical basis to this, as liner wall impedance is finite. The tacit assumption in this is that the shape of the suppression spectrum is more important than its overall effectiveness, so that guidance can be given to liner designers.

Suppression could be limited by formulating the optimization problem with a constraint on suppression area. In that case, the burden of enforcing suppression limits is placed on the optimizer. Another method is to require the suppression spectrum to have an inherently constant area. Statistical probability density functions can be used to characterize families of suppression curves, all having constant areas. Probability density functions inherently have constant enclosed areas, since the probability measure of an entire sample space is unity. Using modified probability density functions naturally constrains the problem so that an optimizer can perform a simpler, unconstrained optimization. Probability density functions can be combined and modified to form quite complex shapes.

\section{Objective Function}

One possible community noise objective function is defined as

$$
O(\mathbf{x})=\sum_{i=1}^{I} w_{i} L_{E P N i}(\mathbf{x})+R_{j} \max \left[0, \frac{A_{i n}(\mathbf{x})+A_{e x}(\mathbf{x})}{A_{i n, \max }+A_{e x, \max }}-1\right]^{2}
$$

$L_{E P N}$ is the notation for the EPNL certification noise metric. As written, $O(\mathbf{x})$ is a composite objective function consisting of the weighted sum of several EPNLs. ${ }^{*}$ In the special case where the weighting factors $w_{i}$ are unity and the number of observers $I=3, O(\mathbf{x})$ can represent the cumulative EPNL if the lateral, flyover, and approach certification monitors are properly located and modeled. Depending on designer's intent, $L_{E P N}$ may consist of suppressed sources only, or other unsuppressed noise sources on the airplane may also be added. When unsuppressed noise sources contribute to $L_{E P N}$, the relative contribution of the suppressed source is diminished, but it may more realistically portray the correct system noise.

The second term is an exterior additive dynamic penalty that penalizes infeasible solutions and drives the final result towards feasible space. $A_{i n}(\mathbf{x})$ and $A_{e x}(\mathbf{x})$ are the enclosed areas of the inlet and exit suppression spectra,

\footnotetext{
*Alternatively, the problem may be set up as a multiple-objective optimization in $L E P N$, resulting in a Pareto-optimal solution.
}

4 of 12

American Institute of Aeronautics and Astronautics 
respectively, which when added together may not exceed the sum of $A_{i n, \max }$ and $A_{e x, \max }$. In this context, the max operator results in the value of the constraint violation if it is positive, otherwise zero is returned. The penalty coefficient $R$ can be sensitive to and increase with $j$, the number of generations in an evolutionary optimization or with the number of solutions searched in a search-strategy optimization. Thus the severity of the penalty increases with the amount of the violation and with the number of successive iterations. For self-constraining suppression spectra based on probability density functions, the need for a penalty is moot, and the max operator will always return zero.

\section{The OpenMDAO Model}

To facilitate the modeling process, the OpenMDAO (version 0.12) frameworking software is used. OpenMDAO ${ }^{7}$ is an open-source computing environment and frameworking tool developed by NASA for multidisciplinary systems analysis and optimization. OpenMDAO is coded in the Python scripting language. Assemblies, components, drivers, and workflows are classes available in OpenMDAO to create objects. The classes are connected to form a sensible, multidisciplinary analysis of a problem. A collection of intrinsic filewrapping utilities are available for component classes to wrap external codes (in this case, ANOPP). The formatting statement in ANOPP that writes EPNL to its output file is modified to print eight significant figures. The component workflow of the noise model is shown in Figure 4.

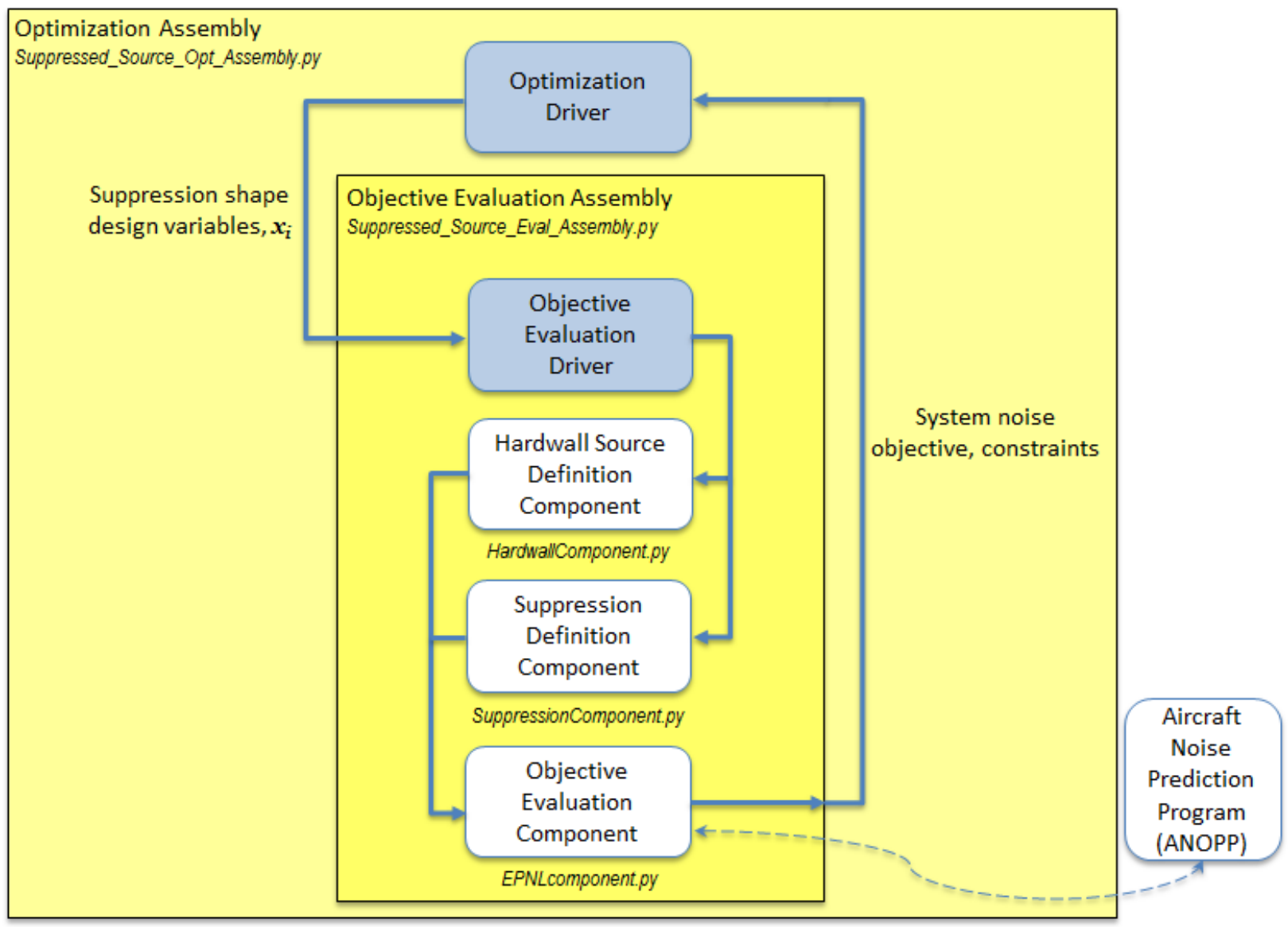

Figure 4. OpenMDAO model component workflow.

The OpenMDAO model consists of two assemblies that may be executed independently. The objective evaluation assembly computes the value of the objective function, given hardwall source and suppression spectra. Inside this assembly, the hardwall source definition component defines the hardwall source spectra to be suppressed. The suppression definition component defines the suppression spectra determined by the independent shape variables, $x_{i}$. Finally, the objective evaluation component computes the suppressed source, applies flight effects, and converts narrowband to one-third octave band spectra. Using filewrapping functions available in OpenMDAO, this component assembles an ANOPP input file from a template, runs it using the suppressed spectra, parses EPNL from the ANOPP 
output, and returns the objective to the assemblies. Thus the most computationally expensive part of the procedure is performed by ANOPP. The outer optimization assembly governs optimizer behavior, manipulation of the $x_{i}$, and calls the objective function evaluation. All of the component analyses are written in native Python code, except of course for the propagation calculations performed externally by ANOPP.

OpenMDAO has drivers that support a variety of optimization methods. Included are classical methods such as gradient-based methods and one direct (search-strategy) method, as well as an evolutionary algorithm. Selecting a successful optimizer is challenging for this kind of problem.

Referring to Figure 2 and the variety of multidisciplinary influences involved, it is clear the objective function is not mathematically smooth. This provides opportunities for gradient-based optimizers to become stuck. Also, if handicapped by an unhelpful starting point, suppression may attack a portion of the source spectrum far from its peak sound pressure level. Due to the logarithmic nature of decibel arithmetic, it is easy for a gradient-based optimizer to gain insufficient purchase on the objective slope and wander. And although probability density functions are recommended above, some of these types of functions exacerbate wandering. Basing a suppression spectrum on the beta probability density function, for example, would be plagued with local minima and search directions would be linked with all of the $x_{i}$ at once, possibly confounding an optimizer. For all of these reasons, it may not be surprising that OpenMDAO's gradient-free Constrained Optimization by Linear Approximation (COBYLA) ${ }^{8}$ driver performed well, at least when independent variables are allowed to be continuous-real.

However, it is not as critical to pinpoint the exact suppression spectrum as it is to simply describe its general shape to an acoustic liner designer. It can be sufficient to cast the spectral shape factors as enumerated discrete variables. Optimization of discrete variables is where evolutionary algorithms excel. Since genetic operators are probabilistic, there is less chance in getting stuck on a suboptimal solution. And if the pun may be pardoned, they are preferred when problems are noisy.

When discrete enumerated variables are chosen, the Pyevolve ${ }^{9}$ evolutionary optimizer is used (although it also performs well when variables are continuous-real). Driver settings are used to configure the process. A constrained tournament selection consisting of crossover and mutation variation operators is used to define each generation. Binary crossovers involve simple exchanges of genes between parent members, while real-parameter crossovers use a Simulated Binary Crossover method. ${ }^{10}$ Random changes are introduced in each generation using real and binary mutation operators. Elite preservation is used to ensure the fittest sample carries on to future generations.

The use of Pyevolve is not without a disadvantage. If allowed to proceed without strict termination criteria, it can be computationally expensive when compared with search-strategy methods. Approaches such as variable-fidelity meta-modeling, or a simplifying reformulation of the problem statement could be applied to reduce computational time. Evolutionary methods are easy to parallelize across multiple compute servers, although that was not done for this task. Pyevolve is used, however, because it is expected to provide good results, largely without regard for its computational efficiency.

\section{Results and Discussion}

The optimization method is applied first to a simple notional source flying over a single observer. The problem is deliberately simplified to verify and validate the process, and to determine the most successful suppression formulations and optimization methods. It is also simple enough so that the optimum solution can be verified by inspection. Afterward, the method is used to derive optimum suppression for a more realistic hardwall fan source using three certification observers.

\section{E. Simple Notional Source}

The simple noise source to be suppressed consists of a narrowband broadband component $L_{b}$ centered at $1000 \mathrm{~Hz}$ combined with a single tone-like structure $L_{t}$ centered at $4900 \mathrm{~Hz}$. These are mathematically represented by a narrowband, frequency-dependent log-parabola and by a modified normal function, respectively (see Eqns. 2, $3^{*}$ and Table 1). The narrowband linear bandwidth of the frequency $f$ is arbitrary but it should be small enough so that there is sufficient detail to adequately represent the source. $L_{b}$ and $L_{t}$ are added decibel-wise to form the total noise source, $L$. The level and frequency of the artificial tone are selected so that a strong correction penalty is assigned to the tonecorrected perceived noise level used in certification. Further, the certification noy weighting procedure emphasizes levels between $1000 \mathrm{~Hz}$ and $10 \mathrm{kHz}$, so $L_{t}$ is accentuated, but $L_{b}$ is not. Thus, on a physical basis, the broadband

\footnotetext{
*The modified normal function has a standard deviation, a5. Although its value is very small compared with the total frequency range, it is not a pure tone. In this sense, it could be looked at as a means to model a bit of tone dispersal, or haystacking. In any case, when coding the source component, it is important to ensure that the peak level $a_{4} / a_{5} / \sqrt{2 \pi}$ is returned when $f$ is near $a_{6}$.
}

6 of 12

American Institute of Aeronautics and Astronautics 
component contains nearly twice as much acoustic power than the tone, but the tone contributes more to certification noise metrics. This noise source is designed strategically to discover how much an optimizer chooses to suppress the tone component relative to the (physically louder) broadband component.

Table 1. Source constants.

\begin{tabular}{c|c}
\hline \hline$a_{1}$ & 100 \\
\hline$a_{2}$ & 30 \\
\hline$a_{3}$ & 1000 \\
\hline$a_{4}$ & 2100 \\
\hline$a_{5}$ & 7 \\
\hline$a_{6}$ & 4900 \\
\hline \hline
\end{tabular}

$$
\begin{aligned}
& L_{b}=a_{1}-a_{2}\left[\log _{10} f / a_{3}\right]^{2} \\
& L_{t}=\frac{a_{4}}{a_{5} \sqrt{2 \pi}} e^{-\left(f-a_{6}\right)^{2} / 2 a_{5}^{2}}
\end{aligned}
$$

The noise source must be referenced to a flight condition, which should be the same reference flight condition used for the suppression spectrum. Although it is somewhat arbitrary, it is convenient to reference them to sea level static conditions. For simplicity, the source is assumed in this problem to have no emission angle dependency. A single observer is used, mimicking an approach certification event with an overflight Mach number of 0.25 at an altitude of 394ft above sea level. Convective amplification and Doppler flight effects impact the source model:

$$
\begin{aligned}
L_{f} & =L-10 k \log _{10}\left(1-M_{f} \cos \theta\right) \\
f_{f} & =f /\left(1-M_{f} \cos \theta\right)
\end{aligned}
$$

where $\theta$ is the polar (yaw) emission angle, referenced to zero at the engine inlet. In flight, levels are adjusted for the flight Mach number $M_{f}$ with a convective amplification term, $k$ (taken to be four for quadrupole emissions). A Doppler term is used to compute shifted flight frequencies, $f_{f}$. Atmospheric absorption and ground reflection calculations are enabled.

For the case when no suppression is applied, the highest tone-weighted perceived noise at the observer occurs at a polar angle of $81.7 \mathrm{deg}$, when the source is at a distance of $402 \mathrm{ft}$. The loudest noise occurs slightly before zenith (and the point of closest approach) due to convection and Doppler effects. Source levels are shown in Figure 5. Narrowband and one-third octave band emitted spectra are

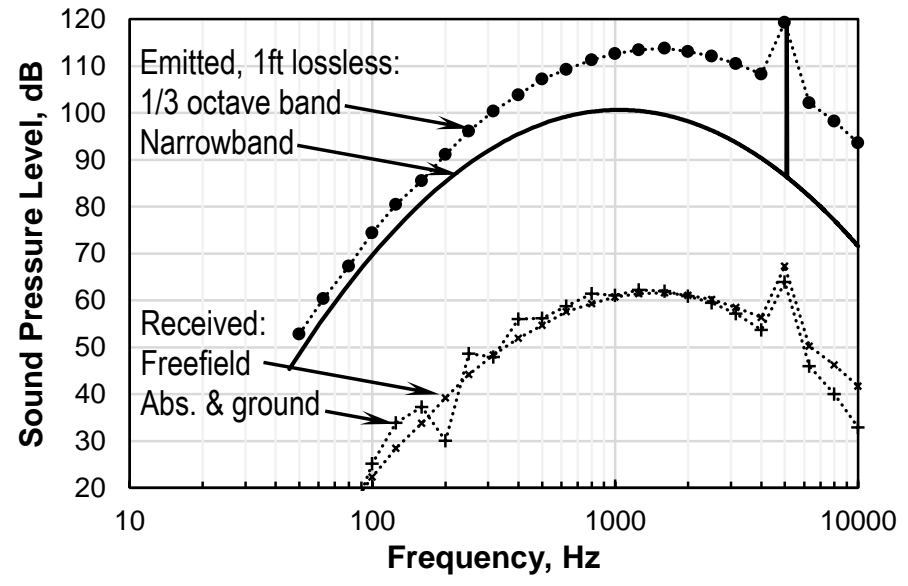

Figure 5. Unsuppressed source at maximum observer noise: emitted vs. received, showing system effects. shown for the lossless source at a distance of one foot. Received one-third octave spectra are also shown, with and without the effects of atmospheric absorption and ground reflections. Absorption begins to have an effect above $1000 \mathrm{~Hz}$, while the influence of reflections can be seen below $800 \mathrm{~Hz}$ where ground effects are most efficient. The EPNL of the unsuppressed source is $80.90 \mathrm{EPNdB}$.

The first type of suppression spectrum $L_{s}$ to be applied is based on the sum of two normal probability density functions:

$$
L_{s}(\mathbf{x})=\frac{C}{\sqrt{2 \pi}}\left[\frac{1}{x_{1}} e^{-\left(f-x_{2}\right)^{2} / 2 x_{1}^{2}}+\frac{1}{x_{3}} e^{-\left(f-x_{4}\right)^{2} / 2 x_{3}^{2}}\right]
$$

$x_{1}$ through $x_{4}$ are the independent shape factor variables that determine the suppression curve levels as a function of frequency. The narrowband linear bandwidth used in Eqn. 6 is the same as the bandwidth used in Eqns. 2 and 3. The model can represent complex suppression shapes with two peaks, perhaps imitating performance of a double degreeof-freedom perforate-over-honeycomb liner. The shape structures can be narrow to suppress a single tone or wide for

7 of 12

American Institute of Aeronautics and Astronautics 
broadband suppression. $C$ is a constant set to 6000: a value large enough to provide on the order of 10EPNdB suppression for a well-performing set of $x_{i}$. Frequency control $\left(x_{2}, x_{4}\right)$ is independent of amplitude control $\left(x_{1}, x_{3}\right)$. $L_{s}$ is taken to be at static conditions and is subtracted from the source before flight effects are applied. The area enclosed by the suppression spectrum is inherently constant, so the optimization is unconstrained.

Since the source consists of just two uncomplicated components in this case, it is logical to simplify the optimization by letting one suppression component attack the broadband source and the other attack the tone. This strategy may not be possible when dealing with a more complex source or with multiple observers where a priori knowledge is difficult to obtain. The frequency range of each suppression component can be controlled by limiting the probability mode variables, $x_{2}$ and $x_{4}$, to the vicinity of the broadband peak and the tone, respectively. Initially, all of the variables are coarsely enumerated. The mode variables $x_{2}$ and $x_{4}$ are set in increments of $100 \mathrm{~Hz}$. The standard deviation variables $x_{1}$ and $x_{3}$ are set in increments of 50Hz. This is Case 1 in the Table 2 summary.

Subsequent cases follow, with each case using insight gained from previous attempts. Case 2 is similar to Case 1, except a finer enumeration of the $x_{i}$ is used. In Case 3, the $x_{i}$ are assumed to be continuous-real with relatively narrow search intervals based on Table 2. Results for simple notional source suppression with Pyevolve.

\begin{tabular}{c|c|c|c|c}
\hline \hline Case & Suppression & $\mathbf{x}$ & Generations & $\boldsymbol{O}(\mathbf{x})$ \\
\hline \hline 1 & Twin-normal & Enumerated, coarse & 300 & 73.217709 \\
2 & Twin-normal & Enumerated, fine & 290 & 73.217522 \\
3 & Twin-normal & Continuous & 150 & 73.210581 \\
4 & Training points & Enumerated & 400 & 72.704830 \\
5 & Training points & Continuous & 800 & 72.654040 \\
\hline \hline
\end{tabular}
the results of Case 2. In the last two cases, the suppression spectrum is defined by a collection of 22 discrete training points connected by a univariate spline curve. A dynamic penalty is added to the objective function to constrain the suppression spectrum to $2 C$ (the area enclosed by Eqn. (6)) so all optimizations can be compared. In these two cases, the frequencies are fixed at critical values determined by a priori knowledge of the system, and the levels are determined by $22 x_{i}$. In Case 4, the $x_{i}$ are enumerated, while in Case 5 they are continuous. In all of the cases, Pyevolve was used in the optimizations with a population of 100 . The optimizations were interrupted when no further improvement in $O(\mathbf{x})$ was thought possible. A post hoc examination of results revealed the running mean and the standard deviation of the populations had become stable long before termination. The COBYLA optimizer was tried on cases where the $x_{i}$ are continuous, with no improvement in the results relative to Pyevolve.

A statistical analysis of preliminary results is helpful in determining subsequent revisions of variable domain limits and enumeration coarseness. In this problem, the variables from the
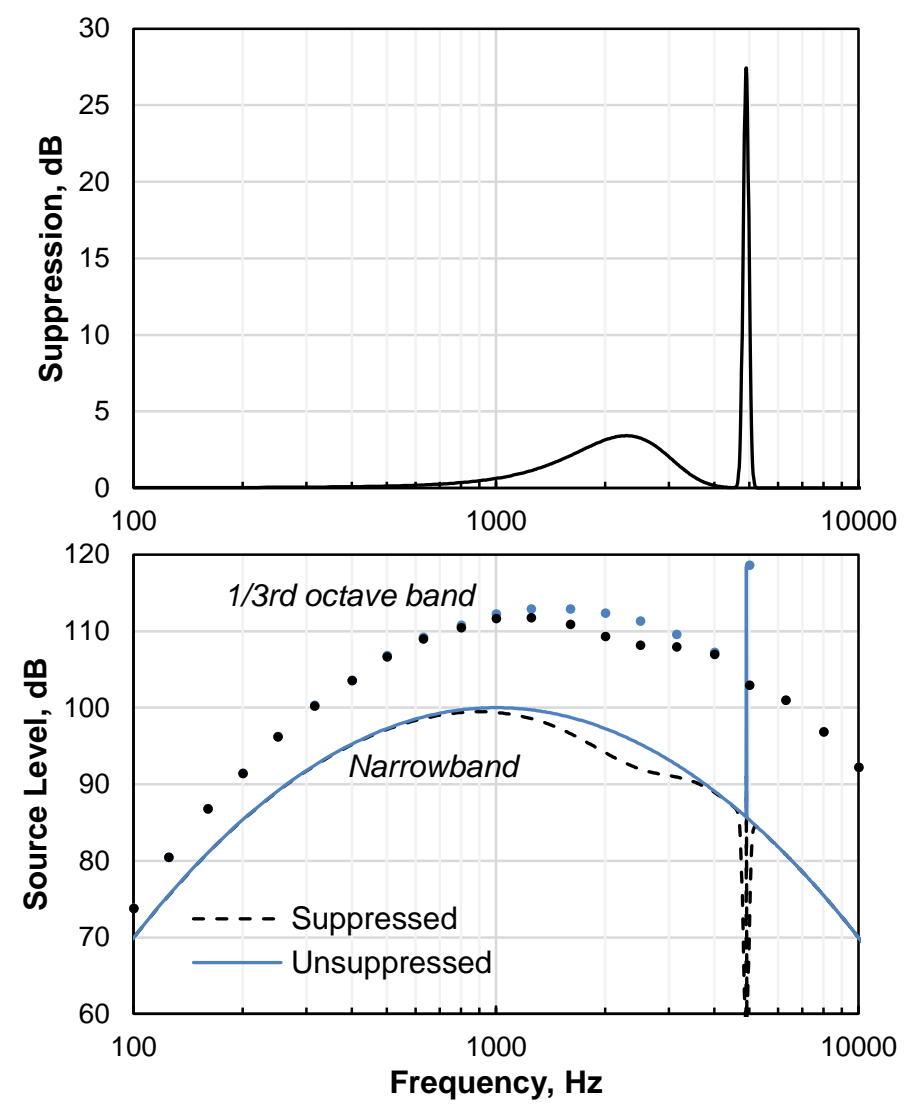

Figure 6. Optimized twin-normal suppression spectrum (top) applied to the notional source (bottom).

evolutionary optimization of Case 1 were analyzed by a frequency of occurrence analysis of the best few thousand samples. For subsequent optimizations, variables were constrained to not stray too far from the "best" $x_{i}$.

The use of training points as suppression is less useful (even though a slightly lower community noise was discovered), since to be set up efficiently, they require some a priori knowledge of the system. This may not always be possible for more complex sources, or when multiple ground observers and engine states are modeled.

8 of 12

American Institute of Aeronautics and Astronautics 
Results of the best optimization using the twin-normal suppression model are shown in Figure 6. The optimal suppression spectrum is shown on the top of the Figure, while the unsuppressed and suppressed lossless emitted spectra on a one-foot arc are shown on the bottom. The first suppression distribution is broadband, with its peak centered on $2289 \mathrm{~Hz}$. The second distribution is centered precisely on the tone at $4900 \mathrm{~Hz}$, but with a much higher peak. To minimize the EPNL, the optimizer chose to emphasize suppressing the tone down to the broadband level by adjusting $x_{3}$, but saw no advantage in suppressing it further. Another interesting result is that the broadband suppression is not centered on the broadband peak of $1000 \mathrm{~Hz}$, but instead at $2289 \mathrm{~Hz}$ : presumably due to the noyweighting process where levels at higher frequencies are emphasized. By inspection, this result suggests the process successfully analyzes multidisciplinary system effects using multiple metrics.

\section{F. Complex Realistic Source}

Next, the method is challenged by a more complex, realistic hardwall fan source, using three certification EPNLs and three engine states. Specifically, experimental acoustic data collected from a scale-model fan test article in NASA's 9- by 15-foot Low Speed Wind Tunnel are used. The fan tested is an 18-blade, 22-inch diameter model of Pratt \& Whitney's Advanced Ducted Propulsor. ${ }^{11}$ With low tip speeds and a design pressure ratio of 1.29 , it is representative of modern, contoured, wide-chord fans used for high-bypass geared turbofans.

The measured noise levels of the fan are adjusted to lossless, freefield, narrowband spectra on a one-foot arc. Data exist for 51 polar (yaw) emission angles ranging from $25 \mathrm{deg}$ to $158 \mathrm{deg}$ from the inlet axis. The source is assumed to be symmetric in emission azimuthal (roll) angle. The levels are adjusted from model-scale, 22-inch fan diameter to full-scale, 88-inch diameter by applying amplitude and frequency shifts for a linear scale factor of 4 . The data are further adjusted from the wind tunnel Mach number of 0.10 to static conditions. Note that using the static condition as a reference state does not imply the grazing flow across the liner surface is also static, since there is a considerable rate of flow inside the inlet. Data at three shaft speeds $(N)$ are considered, representing fan operation at the lateral, flyover and approach flight conditions used in certification.

A mathematical model of static, full-scale, narrowband fan noise is developed to aid data manipulation. Using a noise surrogate model in place of measured spectra allows for removal of extraneous or spurious portions of the spectra that are not believed to be genuine fan noise, such as low-frequency airflow scrubbing and echoic facility noise sources. Fan broadband noise and the first five discrete interaction tones are modeled using a frequency-dependent log-parabola and modified normal functions, respectively, as in Eqns. (2) and (3). Small values of the standard deviation $a_{5}$ yield a pure tone, while larger values can represent a dispersed tone or haystacking.

When modeling each spectrum, at least as important as matching the spectral shape is matching the tone-corrected perceived noise level, since it is the metric used to compute certification EPNL. To prepare the surrogate model for use, an optimization process is performed for every spectrum that adjusts the model constants $a_{i}$ such that both objectives are met. A discussion of this process is described in Ref. 12 and is not repeated here. The result is a onefoot lossless narrowband static noise surrogate model of the fan, $L=L(f, \theta, N)$, that can be projected to arbitrary flight conditions using Eqns. (4) and (5). The flight conditions at each certification noise monitor (shown in Table 3) are typical of a narrow-body 737- or A320-class transport. An example of the surrogate modeling for one spectrum is shown in Figure 7.

In a turbofan application, acoustic treatment lining the inlet is of course separate and distinct from treatment lining the bypass duct. Each lining is entitled to its own set of $x_{i}$. For cases such as this, where the measured source is the inlet and exit sources combined, the total noise must be passed through an inlet-exit relative response filter to resolve separate inlet and exit sources. For this problem, the filter suggested in Ref. 13 is used (shown in Figure 8). Alternately, acoustic barrier walls have been used successfully to separate inlet and exit noise in experimental tests. In those cases, inlet and exit noise sources are naturally separated.

9 of 12

American Institute of Aeronautics and Astronautics 


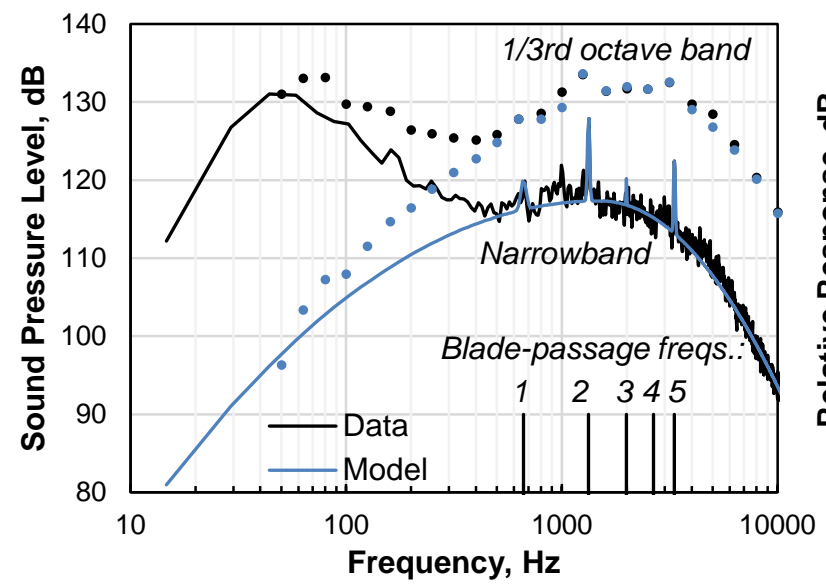

Figure 7. Measurements and surrogate model of scaled, static fan at $100 \% N$, polar angle $90 \mathrm{deg}$.

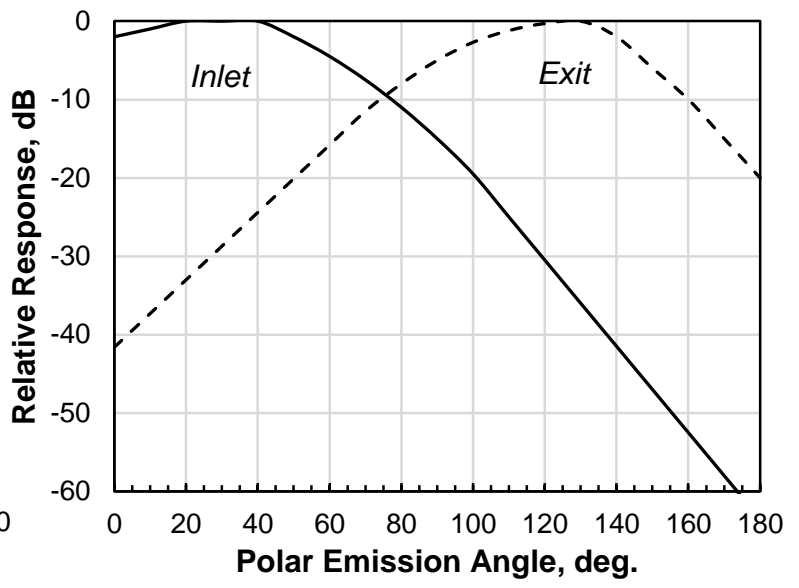

Figure 8. Inlet-exit relative response filter, reproduced from Ref. 13.

Normal probability distribution functions are used to model inlet and exit suppression. Suppression based on two normal functions is used for the inlet (Eqn. 7; suggesting double degree-offreedom treatment), while the exit suppression is based on only one normal function (Eqn. 8; Table 3. Flight conditions. suggesting single degree-of-freedom treatment). This is representative of a perforate-over-honeycomb liner arrangement often found in many modern commercial turbofans. Referring to Ref. 11, it was found that acoustic treatment reduced hardwall noise levels by about 5EPNdB. Thus, the constant $C$ is taken to be 15,000: a value that provides on the order of 5EPNdB suppression for a well-performing set of $x_{i}$. The narrowband linear bandwidth used in Eqns. 7 and 8 is the same as the bandwidth used in the fan source model. Using experience gained from the simple notional source problem, the $x_{i}$ are enumerated and Pyevolve is used with a population size of 100 . The mode variables $x_{2}, x_{4}$ and $x_{6}$ are set in increments of $200 \mathrm{~Hz}$, and the standard deviation variables $x_{1}, x_{3}$ and $x_{5}$ are set in increments of $100 \mathrm{~Hz}$. Variable domain limits are set much further apart than in the simple source problem.

$$
\begin{aligned}
& L_{s, i n}(\mathbf{x})=\frac{C}{\sqrt{2 \pi}}\left[\frac{1}{x_{1}} e^{-\left(f-x_{2}\right)^{2} / 2 x_{1}^{2}}+\frac{1}{x_{3}} e^{-\left(f-x_{4}\right)^{2} / 2 x_{3}^{2}}\right] \\
& L_{s, e x}(\mathbf{x})=\frac{2 C}{x_{5} \sqrt{2 \pi}} e^{-\left(f-x_{6}\right)^{2} / 2 x_{5}^{2}}
\end{aligned}
$$

Once again, $O(\mathbf{x})$ is defined by Eqn. (1). It is a composite objective function consisting of the sum of three EPNLs, where the weighting factors $w_{i}$ are unity. It is representative of a cumulative EPNL. However, no other noise sources are added, so it is not a genuine noise certification prediction. Adding other unsuppressed noise sources such as jet noise or airframe noise would result in a different optimization.

The optimization was interrupted after 500 generations (several days on a contemporary office computer) when it appeared no further improvement in $O(\mathbf{x})$ was thought possible. Afterwards it was observed the running mean and the standard deviation of the populations had stabilized. The optimum suppression spectra and objective function results are shown in Figure 9 and in Table 4. The peak optimum inlet and exit suppression is centered on frequencies between $1 \mathrm{kHz}$ and $2 \mathrm{kHz}$. The inlet spectrum, with its additional degree of freedom, has its second, smaller peak centered on $4 \mathrm{kHz}$.

10 of 12

American Institute of Aeronautics and Astronautics 
Little suppression exists beyond $4 \mathrm{kHz}$ in the exit or beyond $7 \mathrm{kHz}$ in the inlet. Less fan noise is present at those frequencies, so perhaps less suppression is required there, but another reason is related to atmospheric absorption. In the lateral and flyover cases, much greater distances are involved than in the approach case. Absorption plays a larger role at greater distances. For the flyover case, where the point of closest approach is $2400 \mathrm{ft}$, absorption attenuates sound by approximately $60 \mathrm{~dB}$ at $10 \mathrm{kHz}$. Thus, highfrequency fan noise is already very effectively attenuated by the atmosphere. For flyover and lateral noise at least, a liner need not place much emphasis on high frequencies, despite the sensitive noy weighting in that regime.

In more complex cases like this, where there are three ground observers, changing engine state, changing flight condition and dependency on emission angle, it is not always intuitive where suppression should be targeted. Unlike the notional source problem considered first, it is more difficult to verify this solution by simple inspection. In some sense, the optimizer may have to be trusted to have found the best solution.

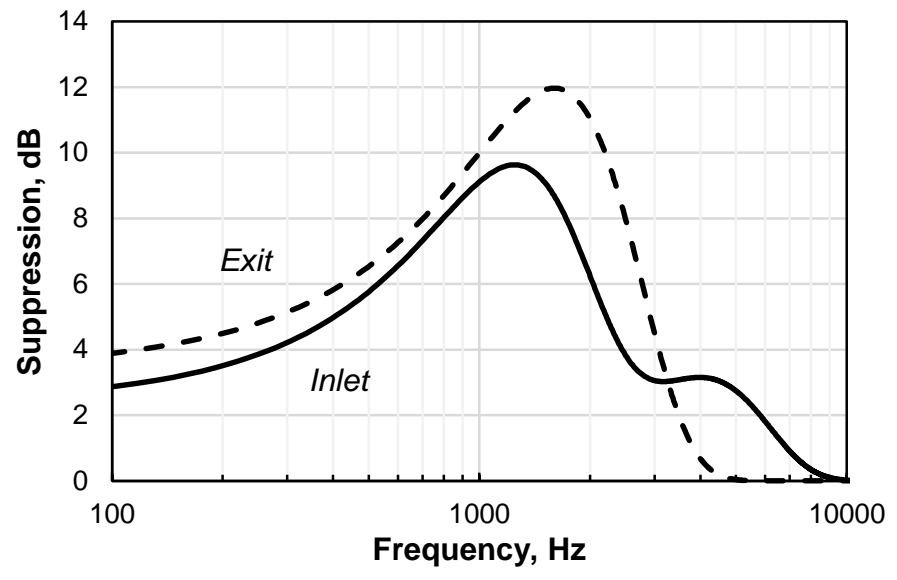

Figure 9. Optimized suppression spectra of inlet and exit treatment applied to a hardwall fan noise source.

Table 4. Optimized results for complex source, EPNdB.

\begin{tabular}{c|c|c}
\hline \hline Monitor & Unsuppressed & Suppressed \\
\hline \hline Lateral & 87.70 & 80.71 \\
Flyover & 80.22 & 72.92 \\
Approach & 89.53 & 83.75 \\
Cumulative & 257.45 & 237.38 \\
\hline \hline
\end{tabular}

\section{Conclusions}

Given a hardwall noise source to suppress with acoustic treatment, there exists an ideal suppression spectrum shape that minimizes noise perceived by observers on the ground. But characteristics of that spectrum can be difficult to know. An analytical method is developed that derives the shape characteristics of the ideal target attenuation spectrum. The method requires mathematically parameterizing the suppression spectrum such that it is represented by a set of independent "shape factors," which are design variables manipulated by an optimizer. Once the ideal shape is known, the geometric design and the impedance characteristics of a real acoustic liner can be derived to match it. The method is written using the OpenMDAO frameworking software developed by NASA for multidisciplinary systems analysis and optimization. Presented in this paper is a description of the method and two test problems. The first problem is made deliberately simple to verify and validate the process, and to determine the most successful suppression formulations and optimization methods. The second problem is the derivation of optimum suppression for a realistic, changing hardwall fan source considering three certification observers.

\section{Acknowledgments}

Thanks to NASA's Advanced Air Transport Technology Project for supporting this study.

\section{References}

${ }^{1}$ Parrott, T.; and Jones, M.: “Assessment of NASA's Aircraft Noise Prediction Capability, Chapter 6: Uncertainty in Acoustic Liner Impedance Measurement and Prediction,” NASA/TP-2012-215653.

${ }^{2}$ International Standards and Recommended Practices - Environmental Protection, "Annex 16 to the Convention on International Civil Aviation, Volume I: Aircraft Noise," $7^{\text {th }}$ Edition, International Civil Aviation Organization (ICAO), Montreal, Canada, July 2014.

${ }^{3}$ U.S. Code of Federal Regulations, Title 14, Chapter I, Part 36, "Noise standards: Aircraft Type and Airworthiness Certification."

${ }^{4}$ Gillian, R.E.: “Aircraft Noise Prediction Program User's Manual,” NASA TM-84486, 1983.

11 of 12

American Institute of Aeronautics and Astronautics 
${ }^{5}$ Zorumski, W.E.: “Aircraft Noise Prediction Program Theoretical Manual, Parts 1 and 2,” NASA TM-83199, 1982; Currently maintained at NASA Langley by the ANOPP team in electronic format and provided upon request; Latest revision: Level 31.

${ }^{6}$ International Electrotechnical Commission, "Octave, Half-Octave, and Third Octave Band Filters Intended for the Analysis of Sounds and Vibrations," IEC Publication No. 225-1966, 1966.

${ }^{7}$ Gray, J., Moore, K.T., Hearn, T.A., and Naylor, B.A., "Standard Platform for Benchmarking Multidisciplinary Design Analysis and Optimization Architectures," AIAA Journal, Vol. 51, No. 10, Oct 2013, pp. 2380-2394.

8Powell, M.J.D.: "A Direct Search Optimization Method that Models the Objective and Constraint Functions by Linear Interpolation", Advances in Optimization and Numerical Analysis, eds. S. Gomez and J-P. Hennart, Kluwer Academic (Dordrecht), 1994, pp. 51-67.

${ }^{9}$ Perone, C.S.: "Pyevolve: A Python Open-Source Framework for Genetic Algorithms," SIGEVOlution, vol. 4, no. 1, pp. 12$20,2009$.

${ }^{10}$ Deb, K.; and Agrawal, R. B.: "Simulated Binary Crossover for Continuous Search Space," Complex Systems, vol. 9, no. 2, pp. 115-148, 1995.

${ }^{11}$ Dittmar, J.H.; Elliott, D.M.; and Bock, L.A.: "Some Acoustic Results from the Pratt and Whitney Advanced Ducted Propulsor - Fan 1,"NASA TM-1999-209049, 1999.

${ }^{12}$ Berton, J.: "System Noise Prediction of the DGEN 380 Turbofan Engine," manuscript accepted for publication, J. Aircraft, 2016, forthcoming.

${ }^{13}$ Heidmann, M.F.: "Interim Prediction Method for Fan and Compressor Source Noise,” NASA TMX-71763, 1979.

12 of 12

American Institute of Aeronautics and Astronautics 